Gianfranco Casnatio

\title{
Special Ulrich bundles on regular surfaces with non-negative Kodaira dimension
}

Received: 2 July 2019 / Accepted: 23 November 2020 / Published online: 19 January 2021

\begin{abstract}
Let $S$ be a regular surface endowed with a very ample line bundle $\mathcal{O}_{S}\left(h_{S}\right)$. Taking inspiration from a very recent result by D. Faenzi on $K 3$ surfaces, we prove that if $\mathcal{O}_{S}\left(h_{S}\right)$ satisfies a short list of technical conditions, then such a polarized surface supports special Ulrich bundles of rank 2. As applications, we deal with general embeddings of regular surfaces, pluricanonically embedded regular surfaces and some properly elliptic surfaces of low degree in $\mathbb{P}^{N}$. Finally, we also discuss about the size of the families of Ulrich bundles on $S$ and we inspect the existence of special Ulrich bundles on surfaces of low degree.
\end{abstract}

\section{Introduction}

Let $\mathbb{P}^{N}$ be the projective space of dimension $N$ over an algebraically closed field $k$ of characteristic 0 . If $X \subseteq \mathbb{P}^{N}$ is a variety, i.e. an integral closed subscheme, then it is naturally endowed with the very ample line bundle $\mathcal{O}_{X}\left(h_{X}\right):=\mathcal{O}_{\mathbb{P}^{N}}(1) \otimes \mathcal{O}_{X}$. We say that a sheaf $\mathcal{E}$ on $X$ is Ulrich (with respect to $\mathcal{O}_{X}\left(h_{X}\right)$ ) if

$$
h^{i}\left(X, \mathcal{E}\left(-i h_{X}\right)\right)=h^{j}\left(X, \mathcal{E}\left(-(j+1) h_{X}\right)\right)=0,
$$

for each $i>0$ and $j<\operatorname{dim}(X)$.

Ulrich bundles on a variety $X$ have many properties: we refer the interested reader to [23], where the authors also raised the following questions.

Questions. Is every variety (or even scheme) $X \subseteq \mathbb{P}^{N}$ the support of an Ulrich sheaf? If so, what is the smallest possible rank for such a sheaf?

When $C$ is a curve, i.e. a smooth variety of dimension 1 , the above questions have very easy answers: indeed if $g$ is the genus of $C$ and $\mathcal{L} \in \mathrm{Pic}^{g-1}(C)$ satisfies $h^{0}(C, \mathcal{L})=0$, then $\mathcal{L}\left(h_{C}\right)$ is an Ulrich line bundle.

At present, no general answers to the above questions are known when $X$ has dimension greater than 1 , though a great number of partial results have been proved: without any claim of completeness, we recall $[2,9,13,14,18,22,24-26,31,32,38$, 39]. The interested reader can also refer to the recent survey [10] for further results.

The author is a member of GNSAGA group of INdAM and is supported by the framework of the MIUR grant Dipartimenti di Eccellenza 2018-2022 (E11G18000350001).

Gianfranco Casnati: Dipartimento di Scienze Matematiche, Politecnico di Torino, c.so Duca degli Abruzzi 24, 10129 Turin, Italy. e-mail: gianfranco.casnati@polito.it

Mathematics Subject Classification: Primary: 14J60; Secondary: 14J27 · 14J28 · 14J29 
In this paper we study the case of surfaces, i.e. smooth varieties of dimension 2 . In particular, following the argument used by D. Faenzi for $K 3$ surfaces in [24] we deal with surfaces $S$ with $q(S)=0$, partially extending analogous results proved in $[9,13,14]$ when $q(S)=p_{g}(S)=0$.

In order to state our main result, we quickly recall a few facts and definitions. Recall that a coherent sheaf $\mathcal{G}$ on $S$ is called simple if $\operatorname{Hom}_{S}(\mathcal{G}, \mathcal{G}) \cong k$. There exists a (possibly non-separated) coarse moduli space $\operatorname{Spl}_{S}\left(r ; c_{1}, c_{2}\right)$ parameterizing simple coherent torsion-free sheaves on $S$ with fixed rank $r$ and Chern classes $c_{1}, c_{2}$ (see [1]).

Moreover, if we set $\mu(\mathcal{G}):=c_{1}(\mathcal{G}) h_{S} / \operatorname{rk}(\mathcal{G})$, the coherent torsion-free sheaf $\mathcal{G}$ is $\mu$-stable (with respect to $\mathcal{O}_{S}\left(h_{S}\right)$ ) if $\mu(\mathcal{K})<\mu(\mathcal{G})$ for each subsheaf $\mathcal{K}$ with $0<\operatorname{rk}(\mathcal{K})<\operatorname{rk}(\mathcal{G})$. There exists a quasi-projective scheme $M_{S}\left(r ; c_{1}, c_{2}\right)$ parameterizing $\mu$-stable coherent torsion-free sheaves on $S$ with fixed rank $r$ and Chern classes $c_{1}, c_{2}$. Such a $M_{S}\left(r ; c_{1}, c_{2}\right)$ can be identified with an open subset of $\mathrm{Spl}_{S}\left(r ; c_{1}, c_{2}\right)$.

An Ulrich bundle $\mathcal{E}$ of rank 2 on a surface $S$ is called special if

$$
c_{1}(\mathcal{E})=c_{1}^{s p}:=3 h_{S}+K_{S},
$$

$K_{S}$ being a canonical divisor. Special Ulrich bundles are easier to construct than arbitrary Ulrich bundles. Indeed, the Serre duality implies that a rank 2 bundle $\mathcal{E}$ with $c_{1}(\mathcal{E})=c_{1}^{s p}$ is Ulrich if and only if

$$
h^{0}\left(S, \mathcal{E}\left(-h_{S}\right)\right)=h^{1}\left(S, \mathcal{E}\left(-h_{S}\right)\right)=0,
$$

Moreover, when $\mathcal{E}$ is a special Ulrich bundle, it is easy to check that

$$
c_{2}(\mathcal{E})=c_{2}^{s p}:=\frac{5 h_{S}^{2}+3 h_{S} K_{S}}{2}+2 \chi\left(\mathcal{O}_{S}\right) .
$$

Finally recall that a line bundle $\mathcal{L}$ is non-special if $h^{1}(S, \mathcal{L})=0$.

We are now able to state the main result of the paper.

Theorem 1.1. Let $S$ be a surface with $q(S)=0$ and $p_{g}(S) \geq 1$, endowed with a very ample non-special line bundle $\mathcal{O}_{S}\left(h_{S}\right)$. Assume $h^{0}\left(S, \mathcal{O}_{S}\left(2 K_{S}-h_{S}\right)\right)=0$.

If $h_{S}^{2}+4 \geq h_{S} K_{S}$, then $S$ supports special simple Ulrich bundles $\mathcal{E}$ of rank 2. If $h_{S}^{2}>h_{S} K_{S}$ and the complement $S_{0}$ of the union of smooth rational curves is dense in $S$, then the aforementioned bundle $\mathcal{E}$ can be chosen $\mu$-stable.

The point corresponding to $\mathcal{E}$ in either $\operatorname{Spl} l_{S}\left(2 ; c_{1}^{s p}, c_{2}^{s p}\right)$ or $M_{S}\left(2 ; c_{1}^{s p}, c_{2}^{s p}\right)$ satisfies $\operatorname{dim} \operatorname{Ext}_{S}^{2}(\mathcal{E}, \mathcal{E})=p_{g}(S)$, is smooth and lies in a unique component of dimension $h_{S}^{2}-K_{S}^{2}+5 \chi(S)$.

Before dealing with the structure of the paper we make some comments about the hypothesis in the statement above.

If $p_{g}(S)=0$ the existence of special Ulrich bundles on $S$ has been proved with the same construction in [13] without the hypothesis $h^{0}\left(S, \mathcal{O}_{S}\left(2 K_{S}-h_{S}\right)\right)=0$.

Nevertheless, the proof of the $\mu$-stability of $\mathcal{E}$ given here cannot be extended to the case $p_{g}(S)=0$ when the Kodaira dimension $\kappa(S)$ is negative, because it rests on the hypothesis $S_{0} \neq \emptyset$, which necessarily imply $\kappa(S) \geq 0$. 
The $\mu$-stability of $\mathcal{E}$ when $\kappa(S)=-\infty$ has been proved in [14] with a different argument, under the additional hypothesis that $k$ is uncountable.

A priori, it could happen that $S_{0}$ is not open even when $\kappa(S) \geq 0$. E.g., in [5] the authors give an example of a $K 3$ surface containing infinitely many smooth rational curves.

In particular, we cannot deduce the $\mu$-stability of $\mathcal{E}$ without further information. E.g. if $S$ is a surface of general type, i.e. $\kappa(S)=2$, then $S$ contains at most a finite number of smooth rational curves by [36], hence $S_{0}$ is certainly dense in this case.

Finally, the vanishing $h^{0}\left(S, \mathcal{O}_{S}\left(2 K_{S}-h_{S}\right)\right)=0$ is fullfilled if $\kappa(S) \leq 1$, thanks to [34, Corollary 2.2.7]. If $\kappa(S)=2$ this is no longer true (see e.g. Example 8.4).

We now deal with the content of the paper. In Sect. 5 we prove the above theorem via a finite induction. We first construct a vector bundle of rank 2 such that $c_{1}(\mathcal{F})=h_{S}+K_{S}, h^{1}(S, \mathcal{F})=0$ and $h^{0}(S, \mathcal{F})=p_{g}(S)$ using standard techniques (see Sect. 3). Then, via a classical result due to I.V. Artamkin (see [3]), we construct inductively a sequence of vector bundles $\mathcal{F}_{d}$ of rank 2 with $1 \leq d \leq p_{g}(S)$ such that $c_{1}\left(\mathcal{F}_{d}\right)=c_{1}(\mathcal{F}), h^{1}\left(S, \mathcal{F}_{d}\right)=0$ and $h^{0}\left(S, \mathcal{F}_{d}\right)=p_{g}(S)-d$ (see Sect. 4). As pointed out above, it follows that $\mathcal{E}:=\mathcal{F}_{p_{g}(S)}\left(h_{S}\right)$ is a special Ulrich bundle of rank 2: in the paper we show that it has all the properties listed in the statement of Theorem 1.1.

It is natural to ask whether surfaces as in Theorem 1.1 actually exist. On the one hand, each surface $S$ with $q(S)=0$ can be endowed with many very ample line bundles satisfying the hypothesis of Theorem 1.1 (see Example 6.1). On the other hand, there are several interesting polarized surfaces satisfying the hypothesis of Theorem 1.1 besides the case of $K 3$ surfaces described in [24]. For instance, each regular surface of general type $S \subseteq \mathbb{P}^{N}$ with $\mathcal{O}_{S}\left(h_{S}\right) \cong \mathcal{O}_{S}\left(n K_{S}\right)$ for an $n \geq 3$ (see Example 6.2). Some properly elliptic surfaces in $\mathbb{P}^{4}$ provide a second interesting example (see Example 6.4).

In Sect. 7 we make some comments about the size of families of Ulrich bundles on the surfaces described in Examples 6.1, 6.2 and 6.4.

Finally, in Sect. 8, we deal with surfaces of low degree in $\mathbb{P}^{N}$, extending some results from [15].

\section{Some preliminary facts}

In this section we list some results which will be used in the paper. For all the other necessary results we refer the reader to [28].

If $\mathcal{G}$ and $\mathcal{H}$ are coherent sheaves on the surface $S$, then the Serre duality holds

$$
\operatorname{Ext}_{S}^{i}\left(\mathcal{H}, \mathcal{G}\left(K_{S}\right)\right) \cong \operatorname{Ext}_{S}^{2-i}(\mathcal{G}, \mathcal{H})^{\vee},
$$

(see [29, Proposition 7.4]: see also [6]). Thus $q(S):=h^{1}\left(S, \mathcal{O}_{S}\right)=h^{1}\left(S, \mathcal{O}_{S}\left(K_{S}\right)\right)$, $p_{g}(S):=h^{2}\left(S, \mathcal{O}_{S}\right)=h^{0}\left(S, \mathcal{O}_{S}\left(K_{S}\right)\right)$ and $h^{2}\left(S, \mathcal{O}_{S}\left(K_{S}\right)\right)=h^{0}\left(S, \mathcal{O}_{S}\right)=1$.

Assume that $\mathcal{H}$ is torsion-free. Then there is a natural injective morphism $\mathcal{O}_{S}\left(K_{S}\right) \rightarrow \mathcal{H} \operatorname{Hom}_{S}\left(\mathcal{H}, \mathcal{H}\left(K_{S}\right)\right)$. Thus the map induced on cohomology

$$
H^{0}\left(S, \mathcal{O}_{S}\left(K_{S}\right)\right) \rightarrow \operatorname{Hom}_{S}\left(\mathcal{H}, \mathcal{H}\left(K_{S}\right)\right) \cong \operatorname{Ext}_{S}^{2}(\mathcal{H}, \mathcal{H})^{\vee}
$$


is injective too. In particular

$$
\operatorname{dim} \operatorname{Hom}_{S}\left(\mathcal{H}, \mathcal{H}\left(K_{S}\right)\right)=\operatorname{dim} \operatorname{Ext}_{S}^{2}(\mathcal{H}, \mathcal{H}) \geq p_{g}(S)
$$

If $\mathcal{G}$ is a vector bundle on $S$, then the Riemann-Roch theorem for $\mathcal{G}$ on $S$ is

$$
h^{0}(S, \mathcal{G})+h^{2}(S, \mathcal{G})=h^{1}(S, \mathcal{G})+\operatorname{rk}(\mathcal{G}) \chi\left(\mathcal{O}_{S}\right)+\frac{c_{1}(\mathcal{G})\left(c_{1}(\mathcal{G})-K_{S}\right)}{2}-c_{2}(\mathcal{G})
$$

We finally recall the Cayley-Bacharach construction of vector bundles on $S$. Let $Z \subseteq S$ be a 0 -dimensional locally complete intersection subscheme and let $\mathcal{L} \in$ $\operatorname{Pic}(S)$. Recall that $Z$ satisfies the Cayley-Bacharach condition with respect to $\mathcal{L}$ if

$$
h^{0}\left(S, \mathcal{I}_{Z \mid S} \otimes \mathcal{L}\right)=h^{0}\left(S, \mathcal{I}_{Z^{\prime} \mid S} \otimes \mathcal{L}\right)
$$

for each subscheme $Z^{\prime} \in Z$ with $\operatorname{deg}\left(Z^{\prime}\right)=\operatorname{deg}(Z)-1$.

Theorem 2.1. Let $S$ be a surface and let $Z \subseteq S$ be a 0 -dimensional locally complete intersection subscheme.

Then there exists a vector bundle $\mathcal{F}$ of rank 2 fitting into an exact sequence of the form

$$
0 \longrightarrow \mathcal{O}_{S} \longrightarrow \mathcal{F} \longrightarrow \mathcal{I}_{Z \mid S} \otimes \mathcal{L} \longrightarrow 0
$$

if and only if $Z$ has the Cayley-Bacharach property with respect to $\mathcal{L}\left(K_{S}\right)$.

Proof. See [30, Theorem 5.1.1].

We close this section with a remark about the locus of smooth rational curves on a surface $S$.

Definition 2.2. If $S$ is a surface, we denote by $\mathcal{D}$ the set of smooth rational curves $D \subseteq S$ and we set $S_{0}:=S \backslash \bigcup_{D \in \mathcal{D}} D$.

Remark 2.3. Notice that if $\kappa(S)=-\infty$, then $S_{0}=\emptyset$. On the opposite side, in the case we are interested in, i.e. $\kappa(S) \geq 0$, then $S_{0}$ is the complement of a countable union of proper closed subschemes.

To prove this assertion we first notice that $D^{2} \leq-1$ for each $D \in \mathcal{D}$ when $\kappa(S) \geq 0$ by [7, Proposition III.2.3]. If $A \in|D+\vartheta|$ for some $\mathcal{O}_{S}(\vartheta) \in \operatorname{Pic}^{0}(S)$, then $D A \leq-1$, hence $D$ is a component of $A$. It follows that $D=A$, because they have the same degree with respect to any very ample line bundle on $S$, i.e. the class of each $D \in \mathcal{D}$ in the Néron-Severi group $\operatorname{NS}(S)$ contains exactly one smooth rational curve.

We deduce from the above discussion that $S_{0}$ is certainly non-empty and dense if $k$ is uncountable (see [28, Exercise V.4.15 (c)]). Nevertheless, as pointed out in the introduction, in many cases $S_{0}$ is actually open and non-empty, without any restriction on the cardinality of $k$ (see [36]). 


\section{The base case}

As explained in the introduction, the proof of Theorem 1.1 is by induction. In this section we deal with its base case.

Let $S$ be a surface with $q(S)=0$ and $\mathcal{O}_{S}\left(h_{S}\right)$ a non-special very ample line bundle. If $h^{0}\left(S, \mathcal{O}_{S}\left(K_{S}-h_{S}\right)\right)=0$, then Equality (2.3) yields $h^{0}\left(S, \mathcal{O}_{S}\left(h_{S}\right)\right)=$ $N+1$ where

$$
N:=\frac{h_{S}^{2}-h_{S} K_{S}}{2}+p_{g}(S) .
$$

In particular $\mathcal{O}_{S}\left(h_{S}\right)$ induces an embedding $S \subseteq \mathbb{P}^{N}$.

Let $X$ be any scheme. In what follows $X^{[\overline{N+2]}}$ denotes the Hilbert scheme of 0 -dimensional subschemes of degree $N+2$ inside $X$.

Construction 3.1. Let $S$ be a surface with $q(S)=0$, endowed with a non-special very ample line bundle $\mathcal{O}_{S}\left(h_{S}\right)$. Let $S \subseteq \mathbb{P}^{N}$ be the induced embedding.

Since $S$ is integral and non-degenerate in $\mathbb{P}^{N}$, it follows the existence of an open non-empty subset $\mathcal{Z} \subseteq S^{[N+2]}$ whose points correspond to schemes $Z$ of $N+2$ points in general linear position inside $\mathbb{P}^{N}$. Each scheme $Z$ corresponding to a point in $\mathcal{Z}$ satisfies the hypothesis of Theorem 2.1 with respect to $\mathcal{O}_{S}\left(h_{S}\right)$, hence there is a rank 2 vector bundle $\mathcal{F}$ fitting into

$$
0 \longrightarrow \mathcal{O}_{S}\left(K_{S}\right) \longrightarrow \mathcal{F} \longrightarrow \mathcal{I}_{Z \mid S}\left(h_{S}\right) \longrightarrow 0
$$

Notice that

$$
c_{1}(\mathcal{F})=h_{S}+K_{S}, \quad c_{2}(\mathcal{F})=\frac{h_{S}^{2}+h_{S} K_{S}}{2}+\chi\left(\mathcal{O}_{S}\right)+1 .
$$

By definition $h^{0}\left(S, \mathcal{O}_{S}\left(h_{S}\right)\right)=N+1, h^{0}\left(Z, \mathcal{O}_{Z}\right)=N+2$ and $h^{1}\left(S, \mathcal{O}_{S}\left(h_{S}\right)\right)=0$. Moreover, the choice of $Z$ implies $h^{0}\left(S, \mathcal{I}_{Z \mid S}\left(h_{S}\right)\right)=0$. Thus the cohomology of the exact sequence

$$
0 \longrightarrow \mathcal{I}_{Z \mid S} \longrightarrow \mathcal{O}_{S} \longrightarrow \mathcal{O}_{Z} \longrightarrow 0
$$

tensored by $\mathcal{O}_{S}\left(h_{S}\right)$ and Equality (2.1) yield

$$
\operatorname{dim} \operatorname{Ext}_{S}^{1}\left(\mathcal{I}_{Z \mid S}\left(h_{S}\right), \mathcal{O}_{S}\left(K_{S}\right)\right)=h^{1}\left(S, \mathcal{I}_{Z \mid S}\left(h_{S}\right)\right)=1,
$$

i.e. $\mathcal{F}$ is uniquely determined by $Z$.

Lemma 3.2. Let $S$ be a surface with $\kappa(S) \geq 0$ and $q(S)=0$, endowed with a non-special very ample line bundle $\mathcal{O}_{S}\left(h_{S}\right)$. Assume $h^{0}\left(S, \mathcal{O}_{S}\left(K_{S}-h_{S}\right)\right)=0$.

Then

$$
h^{0}(S, \mathcal{F})=p_{g}(S), \quad h^{0}\left(S, \mathcal{F}\left(-h_{S}\right)\right)=0, \quad h^{1}(S, \mathcal{F})=0
$$

for the vector bundle $\mathcal{F}$ obtained from a scheme $Z$ as in Construction (3.1). 
Proof. Since $h^{0}\left(S, \mathcal{I}_{Z \mid S}\right)=0$ and $h^{0}\left(S, \mathcal{O}_{S}\left(K_{S}-h_{S}\right)\right)=0$, it follows that the cohomology of Sequence (3.2) tensored by $\mathcal{O}_{S}\left(-h_{S}\right)$ implies $h^{0}\left(S, \mathcal{F}\left(-h_{S}\right)\right)=0$.

The vanishing $h^{0}\left(S, \mathcal{I}_{Z \mid S}\left(h_{S}\right)\right)=0$ implies $h^{0}(S, \mathcal{F})=p_{g}(S)$. The equality $h^{2}(S, \mathcal{F})=h^{0}\left(S, \mathcal{F}\left(-h_{S}\right)\right)=0$, the cohomology of Sequence (3.2) and the vanishing $h^{0}\left(S, \mathcal{I}_{Z \mid S}\left(h_{S}\right)\right)=0$ yield the exact sequence

$$
0 \longrightarrow H^{1}(S, \mathcal{F}) \longrightarrow H^{1}\left(S, \mathcal{I}_{Z \mid S}\left(h_{S}\right)\right) \longrightarrow H^{2}\left(S, \mathcal{O}_{S}\left(K_{S}\right)\right) \longrightarrow 0,
$$

hence $h^{1}(S, \mathcal{F})=h^{1}\left(S, \mathcal{I}_{Z \mid S}\left(h_{S}\right)\right)-h^{2}\left(S, \mathcal{O}_{S}\left(K_{S}\right)\right)$. Since $h^{2}\left(S, \mathcal{O}_{S}\left(K_{S}\right)\right)=1$, it follows that $h^{1}(S, \mathcal{F})=0$, thanks to Equality (3.4).

In the next proposition we deal with the properties of the point corresponding to $\mathcal{F}$ in the moduli spaces $\operatorname{Spl}_{S}\left(2 ; c_{1}(\mathcal{F}), c_{2}(\mathcal{F})\right)$ and $M_{S}\left(2 ; c_{1}(\mathcal{F}), c_{2}(\mathcal{F})\right)$.

To this purpose, we denote by $\mathcal{Z}_{0}$ the open subset of $\mathcal{Z}$ of points corresponding to schemes $Z$ such that $h^{0}\left(S, \mathcal{I}_{Z \mid S}\left(K_{S}\right)\right)=0$. Trivially, $\mathcal{Z}_{0} \neq \emptyset$ if $N+2 \geq p_{g}(S)$. It is immediate to check that such an inequality is equivalent to $h_{S}^{2}+4 \geq h_{S} K_{S}$ if $h^{0}\left(S, \mathcal{O}_{S}\left(K_{S}-h_{S}\right)\right)=0$.

Finally, let $\mathcal{Z}_{1}:=S_{0}^{[N+2]} \cap \mathcal{Z}$. As pointed out in Remark $2.3, \mathcal{Z}_{1}$ could be empty, but if $k$ is uncountable and $\kappa(S) \geq 0$, then it is certainly dense inside $S^{[N+2]}$.

Proposition 3.3. Let $S$ be a surface with $q(S)=0$ and $p_{g}(S) \geq 1$, endowed with a non-special very ample line bundle $\mathcal{O}_{S}\left(h_{S}\right)$. Assume $h^{0}\left(S, \mathcal{O}_{S}\left(K_{S}-h_{S}\right)\right)=0$.

Then the following properties hold for the vector bundle $\mathcal{F}$ obtained from a scheme $Z \in \mathcal{Z}$ as in Construction (3.1)

(1) $\mathcal{F}$ is simple.

(2) $p_{g}(S) \leq \operatorname{dim} \operatorname{Ext}_{S}^{2}(\mathcal{F}, \mathcal{F}) \leq p_{g}(S)+h^{0}\left(S, \mathcal{O}_{S}\left(2 K_{S}-h_{S}\right)\right)$ if $h_{S}^{2}+4 \geq h_{S} K_{S}$ and $Z \in \mathcal{Z}_{0}$.

(3) $\mathcal{F}$ is $\mu$-stable if $h_{S}^{2}>h_{S} K_{S}$ and $Z \in \mathcal{Z}_{1}$.

Proof. In order to prove assertion (1), applying $\operatorname{Hom}_{S}(\mathcal{F},-)$ to Sequences (3.3) tensored by $\mathcal{O}_{S}\left(h_{S}\right)$ and (3.2), taking into account of Lemma 3.2 we obtain

$\operatorname{Hom}_{S}(\mathcal{F}, \mathcal{F}) \subseteq \operatorname{Hom}_{S}\left(\mathcal{F}, \mathcal{I}_{Z \mid S}\left(h_{S}\right)\right) \subseteq \operatorname{Hom}_{S}\left(\mathcal{F}, \mathcal{O}_{S}\left(h_{S}\right)\right) \cong H^{0}\left(S, \mathcal{F}\left(-K_{S}\right)\right)$

Tensoring the cohomology of Sequence (3.2) by $\mathcal{O}_{S}\left(-K_{S}\right)$ we obtain

$$
h^{0}\left(S, \mathcal{F}\left(-K_{S}\right)\right) \leq 1+h^{0}\left(S, \mathcal{I}_{Z \mid S}\left(h_{S}-K_{S}\right)\right) .
$$

The choice of $Z$ and the hypothesis $p_{g}(S) \geq 1$ imply

$$
h^{0}\left(S, \mathcal{I}_{Z \mid S}\left(h_{S}-K_{S}\right)\right) \leq h^{0}\left(S, \mathcal{I}_{Z \mid S}\left(h_{S}\right)\right)=0,
$$

hence $\mathcal{F}$ is simple.

Let us prove assertion (2). The choice of $Z$ implies $h^{0}\left(S, \mathcal{I}_{Z \mid S}\left(K_{S}\right)\right)=0$, then the cohomology of Sequence (3.2) tensored by $\mathcal{O}_{S}\left(K_{S}-h_{S}\right)$ yields

$$
h^{0}\left(S, \mathcal{F}\left(K_{S}-h_{S}\right)\right)=h^{0}\left(S, \mathcal{O}_{S}\left(2 K_{S}-h_{S}\right)\right) .
$$


The cohomology of the same exact sequence tensored by $\mathcal{F}\left(-h_{S}\right) \cong \mathcal{F}^{\vee}\left(K_{S}\right)$ returns

$$
\begin{aligned}
\operatorname{dim} \operatorname{Ext}_{S}^{2}(\mathcal{F}, \mathcal{F}) & =h^{0}\left(S, \mathcal{F} \otimes \mathcal{F}^{\vee}\left(K_{S}\right)\right) \\
& \leq h^{0}\left(S, \mathcal{F} \otimes \mathcal{I}_{Z \mid S}\right)+h^{0}\left(S, \mathcal{O}_{S}\left(2 K_{S}-h_{S}\right)\right)
\end{aligned}
$$

The obvious inclusion $\mathcal{F} \otimes \mathcal{I}_{Z \mid S} \subseteq \mathcal{F}$ yields $h^{0}\left(S, \mathcal{F} \otimes \mathcal{I}_{Z \mid S}\right) \leq p_{g}(S)$ thanks to Lemma 3.2, hence

$$
\operatorname{dim} \operatorname{Ext}_{S}^{2}(\mathcal{F}, \mathcal{F}) \leq p_{g}(S)+h^{0}\left(S, \mathcal{O}_{S}\left(2 K_{S}-h_{S}\right)\right) .
$$

Assertion (2) follows by combining Inequality (2.2) with the above inequality.

Let us prove assertion (3). Thanks to [43, Theorem II.1.2.2], if $\mathcal{F}$ is not $\mu$-stable, then there should exist a sheaf $\mathcal{M} \subseteq \mathcal{F}$ of rank 1 such that $\mathcal{F} / \mathcal{M}$ is torsion-free and

$$
\mu(\mathcal{M}) \geq \mu(\mathcal{F})=\frac{h_{S}^{2}+h_{S} K_{S}}{2} .
$$

The sheaf $\mathcal{M}$ is trivially torsion-free and it is also normal (see [43, Lemma II.1.1.16]). Thus it is a line bundle, because it has rank 1 (see [43, Lemmas II.1.1.12 and II.1.1.15]). It follows that $\mathcal{M} \cong \mathcal{O}_{S}(E)$ for some divisor $E$ on $S$.

We have

$$
\left(K_{S}-E\right) h_{S} \leq \frac{h_{S} K_{S}-h_{S}^{2}}{2}<0 .
$$

The Nakai criterion then implies

$$
h^{0}\left(S, \mathcal{O}_{S}\left(K_{S}-E\right)\right)=0,
$$

hence $\mathcal{O}_{S}(E)$ is not contained in the kernel $\mathcal{K} \cong \mathcal{O}_{S}\left(K_{S}\right)$ of the map $\mathcal{F} \rightarrow \mathcal{I}_{Z \mid S}\left(h_{S}\right)$ in Sequence (3.2) and the composition $\mathcal{O}_{S}(E) \subseteq \mathcal{F} \rightarrow \mathcal{I}_{Z \mid S}\left(h_{S}\right)$ is necessarily non-zero. In particular $h^{0}\left(S, \mathcal{I}_{Z \mid S}\left(h_{S}-E\right)\right) \geq 1$, hence there is $A \in\left|h_{S}-E\right|$ through $Z$.

We claim that $A h_{S} \geq N+1$. Assuming the claim, Equality (3.1) yields

$$
\frac{h_{S}^{2}-h_{S} K_{S}}{2}+p_{g}(S)+1 \leq A h_{S}=\left(h_{S}-E\right) h_{S} \leq \frac{h_{S}^{2}-h_{S} K_{S}}{2},
$$

a contradiction. We deduce that a sheaf $\mathcal{M}$ as above does not exist, hence $\mathcal{F}$ is $\mu$-stable.

It remains to prove the claim. To this purpose, let $C_{1}, \ldots, C_{S}$ be the integral components of $A$ intersecting $Z$ and $B$ their union. Since $Z \subseteq S_{0}$, it follows that $p_{a}\left(C_{i}\right) \geq 1$. It is well known that $p_{a}\left(C_{i} \cup C_{j}\right)=p_{a}\left(C_{i}\right)+p_{a}\left(C_{j}\right)+C_{i} C_{j}-1$ for each $i, j \in\{1, \ldots, s\}, i \neq j$ (e.g. see [28, Exercise V.1.3 (c)]): by combining these remarks with an easy induction on $s$ we then deduce

$$
p_{a}(B) \geq \sum_{i=1}^{s} p_{a}\left(C_{i}\right)-s+1 \geq 1 .
$$


On the one hand, the cohomology of

$$
0 \longrightarrow \mathcal{O}_{S}\left(h_{S}-B\right) \longrightarrow \mathcal{O}_{S}\left(h_{S}\right) \longrightarrow \mathcal{O}_{B}\left(h_{B}\right) \longrightarrow 0
$$

yields $h^{1}\left(B, \mathcal{O}_{B}\left(h_{B}\right)\right) \leq h^{2}\left(S, \mathcal{O}_{S}\left(h_{S}-B\right)\right)$. Vanishing (3.5) then implies

$$
\begin{aligned}
h^{2}\left(S, \mathcal{O}_{S}\left(h_{S}-B\right)\right) & =h^{0}\left(S, \mathcal{O}_{S}\left(B-h_{S}+K_{S}\right)\right) \\
& \leq h^{0}\left(S, \mathcal{O}_{S}\left(A-h_{S}+K_{S}\right)\right)=h^{0}\left(S, \mathcal{O}_{S}\left(K_{S}-E\right)\right)=0
\end{aligned}
$$

hence $h^{1}\left(B, \mathcal{O}_{B}\left(h_{B}\right)\right)=0$. Thus the Riemann-Roch theorem for the curve $B$ and Inequality (3.6) above yield $h^{0}\left(B, \mathcal{O}_{B}\left(h_{B}\right)\right) \leq B h_{S}$. On the other hand, the curve $B$ is not contained in any hyperplane inside $\mathbb{P}^{N}$, because it contains $Z$, hence $h^{0}\left(B, \mathcal{O}_{B}\left(h_{B}\right)\right) \geq N+1$.

We then deduce that $A h_{S} \geq B h_{S} \geq N+1$, hence the claim is proved and the proof of assertion (3) is complete.

Remark 3.4. Construction 3.1 makes sense also in the case $p_{g}(S)=0$. Indeed it is the method we used in $[13,14]$ for proving the existence of special Ulrich bundles when $p_{g}(S)=q(S)=0$. Notice that in this case the inequality $h_{S}^{2}>h_{S} K_{S}$ is for free.

On the one hand, the proof of Lemma 3.2 can be carried over word by word to this case. On the other hand, the proof of assertion (1) of Proposition 3.3 cannot be extended to the case $p_{g}(S)=0$. Moreover, the proof of assertion (3) is alternative to $\left[13,14\right.$, Theorem 1.2] when $\mathcal{Z}_{1} \neq \emptyset$ : in particular, it certainly needs $\kappa(S) \geq 0$.

One of the hypothesis of $[13,14$, Theorem 1.2] is that $k$ is uncountable. Thus, the above proof and [36] extend such a result when $\kappa(S)=2$ also to the case of a countable base field. When $p_{g}(S)=0$ and $\kappa(S) \leq 1$, as in the case $p_{g}(S) \geq 1$, the condition $\mathcal{Z}_{1} \neq \varnothing$ is not immediate: e.g. there exist Enriques surfaces containing infinitely many rational curves (see [19]).

\section{The inductive step}

In this section we explain the inductive step of the proof of Theorem 1.1.

Construction 4.1. Let $S$ be a surface endowed with a very ample line bundle $\mathcal{O}_{S}\left(h_{S}\right)$. Let $\mathcal{F}$ be a vector bundle of rank 2 such that $h^{0}(S, \mathcal{F}) \geq 1$. Thus for each point $p \in S$, there exist non-zero morphisms $\varphi: \mathcal{F} \rightarrow \mathcal{O}_{p}$.

Each $\varphi$ as above is surjective and we have the exact sequence

$$
0 \longrightarrow \mathcal{F}_{\varphi} \longrightarrow \mathcal{F} \stackrel{\varphi}{\longrightarrow} \mathcal{O}_{p} \longrightarrow 0,
$$

where $\mathcal{F}_{\varphi}:=\operatorname{ker}(\varphi)$. Notice that

$$
c_{1}\left(\mathcal{F}_{\varphi}\right)=c_{1}(\mathcal{F}), \quad c_{2}\left(\mathcal{F}_{\varphi}\right)=c_{2}(\mathcal{F})+1,
$$

because $c_{1}\left(\mathcal{O}_{p}\right)=0$ and $c_{2}\left(\mathcal{O}_{p}\right)=-p$ : see [27, Example 15.3.1]. 
Lemma 4.2. Let $S$ be a surface endowed with a very ample line bundle $\mathcal{O}_{S}\left(h_{S}\right)$. Assume that $\mathcal{F}$ is a vector bundle of rank 2 such that $h^{0}\left(S, \mathcal{F}\left(-h_{S}\right)\right)=0$ and $h^{0}(S, \mathcal{F}) \geq 1$.

If $p \in S$ and $\varphi \in \operatorname{Hom}_{S}\left(\mathcal{F}, \mathcal{O}_{p}\right)$ are general, then

$$
h^{0}\left(S, \mathcal{F}_{\varphi}\right)=h^{0}(S, \mathcal{F})-1, \quad h^{0}\left(S, \mathcal{F}_{\varphi}\left(-h_{S}\right)\right)=0, \quad h^{1}\left(S, \mathcal{F}_{\varphi}\right)=h^{1}(S, \mathcal{F})
$$

for the sheaf $\mathcal{F}_{\varphi}$ obtained from $\mathcal{F}$ as in Construction (4.1).

Proof. The inequality $h^{0}\left(S, \mathcal{F}_{\varphi}\left(-h_{S}\right)\right) \leq h^{0}\left(S, \mathcal{F}\left(-h_{S}\right)\right) \leq 0$ is trivial. The equality $h^{0}\left(S, \mathcal{F}_{\varphi}\right)=h^{0}(S, \mathcal{F})-1$ is equivalent to the surjectivity of the map $\bar{\varphi}: H^{0}(S, \mathcal{F}) \rightarrow k$ induced by $\varphi$. The fact that $\bar{\varphi}$ is surjective for general $p \in S$ and $\varphi \in \operatorname{Hom}_{S}\left(\mathcal{F}, \mathcal{O}_{p}\right)$ is an open property, thus it suffices to check the existence of at least one $p$ and one $\varphi$ with such a property.

To this purpose there exists a non zero $s \in H^{0}(S, \mathcal{F})$. Its zero locus is the union of a 0 -dimensional scheme $X$ and a divisor $E$ on $S$. In particular we have an exact sequence of the form

$$
0 \longrightarrow \mathcal{O}_{S}(E) \stackrel{\varepsilon}{\longrightarrow} \mathcal{F} \longrightarrow \mathcal{I}_{X \mid S}\left(c_{1}(\mathcal{F})-E\right) \longrightarrow 0
$$

Let now $p \notin X \cup E$. Applying $\operatorname{Hom}_{S}\left(\mathcal{I}_{X \mid S}\left(c_{1}(\mathcal{F})-E\right),-\right)$ to the exact sequence

$$
0 \longrightarrow \mathcal{I}_{p \mid S}(E) \longrightarrow \mathcal{O}_{S}(E) \longrightarrow \mathcal{O}_{p} \longrightarrow 0
$$

we obtain the exact sequence

$$
\begin{aligned}
\operatorname{Ext}_{S}^{1}\left(\mathcal{I}_{X \mid S}\left(c_{1}(\mathcal{F})-E\right), \mathcal{I}_{p \mid S}(E)\right) & \stackrel{\delta}{\longrightarrow} \operatorname{Ext}_{S}^{1}\left(\mathcal{I}_{X \mid S}\left(c_{1}(\mathcal{F})-E\right), \mathcal{O}_{S}(E)\right) \\
& \longrightarrow \operatorname{Ext}_{S}^{1}\left(\mathcal{I}_{X \mid S}\left(c_{1}(\mathcal{F})-E\right), \mathcal{O}_{p}\right)
\end{aligned}
$$

We have

$$
\begin{aligned}
& \mathcal{H o m}_{S}\left(\mathcal{I}_{X \mid S}\left(c_{1}(\mathcal{F})-E\right), \mathcal{O}_{p}\right) \cong \mathcal{O}_{p}, \\
& \mathcal{E} x t_{S}^{1}\left(\mathcal{I}_{X \mid S}\left(c_{1}(\mathcal{F})-E\right), \mathcal{O}_{p}\right)=0
\end{aligned}
$$

because we compute them locally on $X$ and $p \notin X \cup E$. The exact sequence of the low degree terms of the spectral sequence

$$
E_{2}^{i, j}:=H^{i}\left(S, \mathcal{E} x t_{S}^{j}\left(\mathcal{I}_{X \mid S}\left(c_{1}(\mathcal{F})-E\right), \mathcal{O}_{p}\right)\right)
$$

then yields $\operatorname{Ext}_{S}^{1}\left(\mathcal{I}_{X \mid S}\left(c_{1}(\mathcal{F})-E\right), \mathcal{O}_{p}\right)=0$. It follows that the map $\delta$ above is surjective, hence we can lift Sequence (4.1) to another exact sequence obtaining a 
commutative diagram of the form

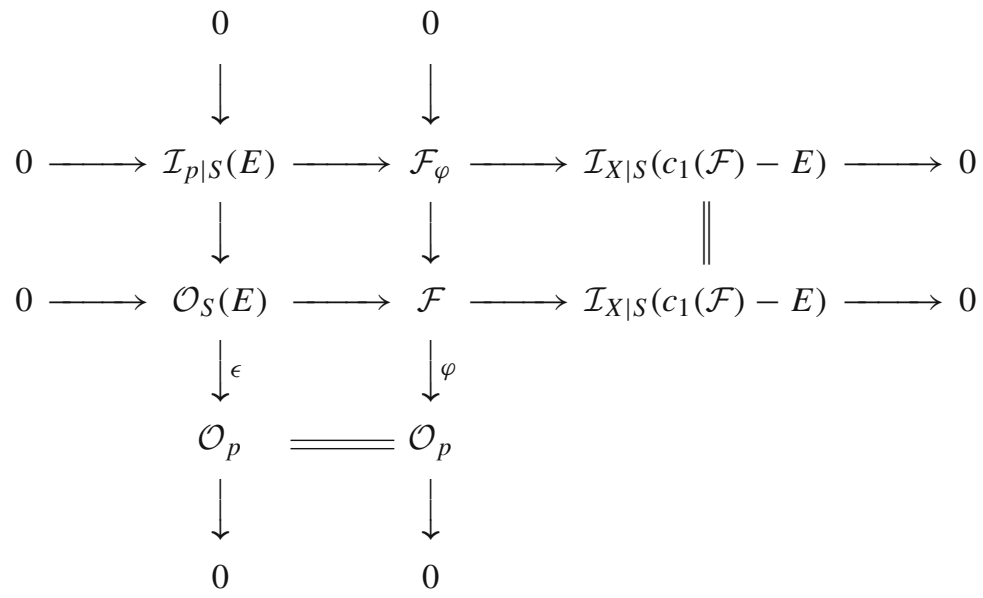

Since $p \notin E$, it follows that $h^{0}\left(S, \mathcal{I}_{p \mid S}(E)\right)=h^{0}\left(S, \mathcal{O}_{S}(E)\right)-1$, hence the map $\bar{\epsilon}: H^{0}\left(S, \mathcal{O}_{S}(E)\right) \rightarrow k$ induced by $\epsilon$ is surjective. The commutativity of the diagram then implies that the same is true for $\bar{\varphi}$. Thus $h^{0}\left(S, \mathcal{F}_{\varphi}\right)=h^{0}(S, \mathcal{F})-1$ and $h^{1}\left(S, \mathcal{F}_{\varphi}\right)=h^{1}(S, \mathcal{F})$ for $\varphi$ general enough.

Proposition 4.3. Let $S$ be a surface endowed with a very ample line bundle $\mathcal{O}_{S}\left(h_{S}\right)$. Assume that $\mathcal{F}$ is a vector bundle of rank 2 with $h^{0}(S, \mathcal{F}) \geq 1$.

Then the following properties hold for a sheaf $\mathcal{F}_{\varphi}$ obtained from $\mathcal{F}$ as in (4.1)

(1) $\mathcal{F}_{\varphi}$ is simple if the same is true for $\mathcal{F}$.

(2) $p_{g}(S) \leq \operatorname{dim}_{\operatorname{Ext}_{S}^{2}}^{2}\left(\mathcal{F}_{\varphi}, \mathcal{F}_{\varphi}\right) \leq \operatorname{dim}_{\operatorname{Ext}_{S}^{2}}^{2}(\mathcal{F}, \mathcal{F})$.

(3) $\mathcal{F}_{\varphi}$ is $\mu$-stable if the same is true for $\mathcal{F}$.

Proof. In order to prove assertion (1), we notice that the quotient map $\mathcal{O}_{S} \rightarrow \mathcal{O}_{p}$ induces by duality an inclusion $0 \neq \operatorname{Hom}_{S}\left(\mathcal{O}_{p}, \mathcal{O}_{p}\right) \subseteq \operatorname{Hom}_{S}\left(\mathcal{O}_{S}, \mathcal{O}_{p}\right) \cong k$, hence

$$
\operatorname{Hom}_{S}\left(\mathcal{O}_{p}, \mathcal{O}_{p}\right) \cong k
$$

Equality (2.1) yields

$$
\operatorname{dim} \operatorname{Ext}_{S}^{i}\left(\mathcal{O}_{p}, \mathcal{F}\right)=h^{2-i}\left(S, \mathcal{F}^{\vee}\left(K_{S}\right) \otimes \mathcal{O}_{p}\right)=2 \delta_{2, i}
$$

Thus the functor $\operatorname{Hom}_{S}\left(\mathcal{O}_{p},-\right)$ applied to Sequence (4.1) we obtain

$$
\operatorname{Ext}_{S}^{1}\left(\mathcal{O}_{p}, \mathcal{F}_{\varphi}\right) \cong \operatorname{Hom}_{S}\left(\mathcal{O}_{p}, \mathcal{O}_{p}\right)
$$

On the one hand, each map in $\operatorname{Hom}_{S}\left(\mathcal{F}, \mathcal{F}_{\varphi}\right)$ induces a map in $\operatorname{Hom}_{S}(\mathcal{F}, \mathcal{F})$ by composing with the inclusion $\mathcal{F}_{\varphi} \subseteq \mathcal{F}$ : it follows that the composed map is never surjective. On the other hand, each non-zero element in $\operatorname{Hom}_{S}(\mathcal{F}, \mathcal{F})$ must be a homothety because $\mathcal{F}$ is simple, hence it is an isomorphism. We deduce that $\operatorname{Hom}_{S}\left(\mathcal{F}, \mathcal{F}_{\varphi}\right)=0$. 
Thus we obtain $\operatorname{Hom}_{S}\left(\mathcal{F}_{\varphi}, \mathcal{F}_{\varphi}\right) \subseteq \operatorname{Ext}_{S}^{1}\left(\mathcal{O}_{p}, \mathcal{F}_{\varphi}\right)$, by applying $\operatorname{Hom}_{S}\left(-, \mathcal{F}_{\varphi}\right)$ to Sequence (4.1). If we combine this inclusion with Equality (4.3) and (4.4), we finally obtain that $\mathcal{F}_{\varphi}$ is simple, i.e. assertion (1) is proven.

We prove assertion (2). Since $\mathcal{F}$ is a vector bundle, the following obvious equalities

$$
h^{0}\left(S, \mathcal{F}^{\vee} \otimes \mathcal{O}_{p}\right)=2, \quad h^{1}\left(S, \mathcal{F}^{\vee} \otimes \mathcal{O}_{p}\right)=h^{2}\left(S, \mathcal{F}^{\vee} \otimes \mathcal{O}_{p}\right)=0
$$

hold, hence $\operatorname{Ext}_{S}^{2}\left(\mathcal{F}, \mathcal{F}_{\varphi}\right) \cong \operatorname{Ext}_{S}^{2}(\mathcal{F}, \mathcal{F})$, by applying $\operatorname{Hom}_{S}(\mathcal{F},-)$ to Sequence (4.1). Equality (2.1) and $\operatorname{Hom}_{S}\left(\mathcal{F}_{\varphi},-\right)$ applied to Sequence (4.1) tensored by $\mathcal{O}_{S}\left(K_{S}\right)$ yield

$$
\begin{aligned}
\operatorname{Ext}_{S}^{2}\left(\mathcal{F}_{\varphi}, \mathcal{F}_{\varphi}\right)^{\vee} \cong \operatorname{Hom}_{S}\left(\mathcal{F}_{\varphi}, \mathcal{F}_{\varphi}\left(K_{S}\right)\right) & \subseteq \operatorname{Hom}_{S}\left(\mathcal{F}_{\varphi}, \mathcal{F}\left(K_{S}\right)\right) \\
& \cong \operatorname{Ext}_{S}^{2}\left(\mathcal{F}, \mathcal{F}_{\varphi}\right)^{\vee} \cong \operatorname{Ext}_{S}^{2}(\mathcal{F}, \mathcal{F})^{\vee} .
\end{aligned}
$$

The statement follows from the above inclusion and Inequality (2.2).

Consider now assertion (3). Since $\mathcal{F}_{\varphi} \subseteq \mathcal{F}$ and $\mu\left(\mathcal{F}_{\varphi}\right)=\mu(\mathcal{F})$, it follows that each subsheaf destabilizing $\mathcal{F}_{\varphi}$ also destabilizes $\mathcal{F}$. Thus assertion (3) is proven.

\section{The proof of Theorem 1.1}

In this section we put together the above results with the following classical theorem for proving Theorem 1.1 stated in the introduction.

Theorem 5.1. Let $S$ be a surface, $p \in S, \mathcal{F}$ a simple vector bundle of rank $r \geq 2$ with $\operatorname{Ext}_{S}^{2}(\mathcal{F}, \mathcal{F})=p_{g}(S), \varphi \in \operatorname{Hom}_{S}\left(\mathcal{F}, \mathcal{O}_{p}\right)$ non-zero and $\mathcal{F}_{\varphi}:=\operatorname{ker}(\varphi)$.

Then $\mathcal{F}_{\varphi}$ has a universal deformation whose general sheaf is locally free at $p$.

Proof. See [3, Theorem 1.4 and Corollary 1.5]. The simplicity hypothesis is mentioned in [3, p. 450].

As pointed out in the Introduction, if $\mathcal{E}$ is a vector bundle on $S$ with first Chern class $c_{1}(\mathcal{E})=3 h_{S}+K_{S}$, then Equality (2.1) implies that $\mathcal{E}$ is Ulrich if and only if

$$
h^{0}\left(S, \mathcal{E}\left(-h_{S}\right)\right)=h^{1}\left(S, \mathcal{E}\left(-h_{S}\right)\right)=0 .
$$

Let $\mathcal{F}$ be the bundle defined in Construction 3.1. If $p_{g}(S) \geq 1$, then $\mathcal{E}:=\mathcal{F}\left(h_{S}\right)$ satisfies all the conditions for being a special Ulrich bundle, but the vanishing $h^{0}\left(S, \mathcal{E}\left(-h_{S}\right)\right)=h^{0}(S, \mathcal{F})=0$. Nevertheless, $\mathcal{F}$ can be viewed as a good approximation of a special Ulrich bundle. For this reason we introduce the following definition.

Definition 5.2. Let $S$ be a surface endowed with a very ample line bundle $\mathcal{O}_{S}\left(h_{S}\right)$. A torsion-free coherent sheaf $\mathcal{F}$ of rank 2 on $S$ is called $d-\operatorname{good}$ if $c_{1}(\mathcal{F})=c_{1}$ where $c_{1}:=h_{S}+K_{S}, h^{0}(S, \mathcal{F})=d$ and $h^{1}(S, \mathcal{F})=h^{0}\left(S, \mathcal{F}\left(-h_{S}\right)\right)=0$. 
As explained above, if $\mathcal{F}$ is a 0 -good bundle, then $\mathcal{E}:=\mathcal{F}\left(h_{S}\right)$ is a special Ulrich bundle of rank 2 .

If $q(S)=0$, then the bundle $\mathcal{F}$ obtained from a scheme $Z$ as in Construction 3.1 is $p_{g}(S)$-good by Lemma 3.2: in particular if $p_{g}(S)=0$, then $\mathcal{F}\left(h_{S}\right)$ is a special Ulrich bundle.

Remark 5.3. Notice that, thanks to Equality (2.3), a vector bundle $\mathcal{F}$ of rank 2 is $d$-good if and only if

$$
c_{1}(\mathcal{F})=c_{1}, \quad c_{2}(\mathcal{F})=c_{2}^{(d)}:=\frac{h_{S}^{2}+h_{S} K_{S}}{2}+2 \chi\left(\mathcal{O}_{S}\right)-d
$$

and $h^{1}(S, \mathcal{F})=h^{0}\left(S, \mathcal{F}\left(-h_{S}\right)\right)=0$, because $h^{2}(S, \mathcal{F})=h^{0}\left(S, \mathcal{F}\left(-h_{S}\right)\right)$.

We are now able to prove Theorems 1.1 stated in the introduction.

Proof of Theorem 1.1.. We will prove the statement by descending induction, showing for each $d=p_{g}(S), \ldots, 0$ the existence of a simple (resp. $\mu$-stable) $d$-good bundle $\mathcal{F}_{d}$ with $\operatorname{dim} \operatorname{Ext}_{S}^{2}\left(\mathcal{F}_{d}, \mathcal{F}_{d}\right)=p_{g}(S)$.

For the base step let $\mathcal{F}_{p_{g}(S)}$ be the bundle $\mathcal{F}$ defined in Construction (3.1) from $Z \in \mathcal{Z}$ (resp. $Z \in \mathcal{Z}_{1}$ ) which is $p_{g}(S)$-good and simple when $h_{S}^{2}+4 \geq h_{S} K_{S}$ (resp. $\mu$-stable when $h_{S}^{2}>h_{S} K_{S}$ ), thanks to Lemma 3.2 and Proposition 3.3.

Now let $p_{g}(S) \geq d \geq 1$ and assume the existence of a simple (resp. $\mu$-stable) $d$-good bundle $\mathcal{F}_{d}$ with $\operatorname{dim} \operatorname{Ext}_{S}^{2}\left(\mathcal{F}_{d}, \mathcal{F}_{d}\right)=p_{g}(S)$. It follows from Construction 4.1 the existence of a simple (resp. $\mu$-stable) $(d-1)$-good sheaf $\mathcal{F}_{\varphi}$ with $\operatorname{dim} \operatorname{Ext}_{S}^{2}\left(\mathcal{F}_{\varphi}, \mathcal{F}_{\varphi}\right)=p_{g}(S)$, thanks to Lemmas 3.2, 4.2 and Proposition 4.3.

Thanks to [40, Theorem 0.3], the point corresponding to $\mathcal{F}_{\varphi}$ in $\operatorname{Spl}_{S}\left(2 ; c_{1}, c_{2}^{d-1}\right)$ (resp. $\left.M_{S}\left(2 ; c_{1}, c_{2}^{d-1}\right)\right)$ is smooth and it lies in a component $M$ of dimension

$$
\operatorname{dim} \operatorname{Ext}_{S}^{1}\left(\mathcal{F}_{\varphi}, \mathcal{F}_{\varphi}\right)=h_{S}^{2}-K_{S}^{2}+5 \chi\left(\mathcal{O}_{S}\right)-4 d+4
$$

Thus we have an integral open smooth neighborhood $B \subseteq M$ of the point corresponding to $\mathcal{F}_{\varphi}$ and a flat family $\mathfrak{F}$ over $B$ with $\mathfrak{F}_{b_{0}} \cong \mathcal{F}_{\varphi}$ for some $b_{0} \in B$.

Since $\mathcal{F}_{d}$ satisfies the hypothesis of Theorem 5.1 and $\mathcal{F}_{\varphi}$ is locally free on $S \backslash\{p\}$, up to shrinking $B$ we can assume that $\mathfrak{F}_{b}$ is locally free at $p$ for each $b \in B \backslash\left\{b_{0}\right\}$. Thus the sheaf $\mathcal{F}_{d-1}:=\mathfrak{F}_{b}$ is a simple (resp. $\mu$-stable) $(d-1)$-good bundle for each choice $b \in B \backslash\left\{b_{0}\right\}$.

The smoothness of $B$ implies that the tangent space at $b \in B$ has dimension

$$
\begin{aligned}
h_{S}^{2}-K_{S}^{2}+4 \chi\left(\mathcal{O}_{S}\right) & -4 d+5+\operatorname{dim} \operatorname{Ext}_{S}^{2}\left(\mathcal{F}_{d-1}, \mathcal{F}_{d-1}\right) \\
& =\operatorname{dim}_{\operatorname{Ext}_{S}^{1}}^{1}\left(\mathcal{F}_{d-1}, \mathcal{F}_{d-1}\right)=\operatorname{dim} \operatorname{Ext}_{S}^{1}\left(\mathcal{F}_{\varphi}, \mathcal{F}_{\varphi}\right),
\end{aligned}
$$

hence $\operatorname{dim} \operatorname{Ext}_{S}^{2}\left(\mathcal{F}_{d-1}, \mathcal{F}_{d-1}\right)=p_{g}(S)$.

We can repeat the above steps untill $d=1$. After $p_{g}(S)$ steps we obtain the existence of a 0 -good bundle $\mathcal{F}_{0}$ representing a smooth point in $S p l_{S}\left(2 ; c_{1}, c_{2}^{\left(p_{g}(S)\right)}\right)$ (resp. $M_{S}\left(2 ; c_{1}, c_{2}^{\left(p_{g}(S)\right)}\right)$ lying in a component of dimension $h_{S}^{2}-K_{S}^{2}+5 \chi\left(\mathcal{O}_{S}\right)$, which is necessarily unique. 
In order to prove the theorem we finally notice that the map $\mathcal{G} \mapsto \mathcal{G}\left(h_{S}\right)$ induces a well defined isomorphism $\operatorname{Spl}_{S}\left(2 ; c_{1}, c_{2}^{\left(p_{g}(S)\right)}\right) \cong \operatorname{Spl}_{S}\left(2 ; c_{1}^{s p}, c_{2}^{s p}\right)($ resp. $\left.M_{S}\left(2 ; c_{1}, c_{2}^{\left(p_{g}(S)\right)}\right) \cong M_{S}\left(2 ; c_{1}^{s p}, c_{2}^{s p}\right)\right)$.

\section{Examples}

In this section we give some applications of Theorem 1.1. We first show that Ulrich bundles are quite common on regular surfaces. Then we give examples of polarized surfaces fulfilling the hypothesis of Theorem 1.1.

Example 6.1. Let $S$ be a surface with $q(S)=0, p_{g}(S) \geq 1$ and $\mathcal{O}_{S}(H)$ an ample line bundle. Then there exists an integer $n_{0}$ such that for each $n \geq n_{0}$ the surface $S$ supports a special Ulrich bundle of rank 2 with respect to $\mathcal{O}_{S}(n H)$. If $\mathcal{Z}_{1} \neq \varnothing$ such bundles can be taken $\mu$-stable.

Indeed, it suffices to check that there is $n_{0}$ such that $\mathcal{O}_{S}(n H)$ satisfies the hypothesis of Theorem 1.1 for $n \geq n_{0}$, i.e. $n^{2} H^{2}>n H K_{S}$ and $h^{0}\left(S, \mathcal{O}_{S}\left(2 K_{S}-\right.\right.$ $n H))=0$.

Since $H$ is ample, it follows that there is $n_{1}$ such that $n_{1} H$ is very ample and non-special. Again the ampleness of $H$ implies $H^{2} \geq 1$, hence there is $n_{2}$ such that $\left(2 K_{S}-n_{2} H\right) H<0$, hence $h^{0}\left(S, \mathcal{O}_{S}\left(2 K_{S}-n_{2} H\right)\right)=0$ by the Nakai criterion. Finally, it is easy to check that for such an $n_{2}$ we also have $\left(n_{2} H-K_{S}\right) H>0$. It then suffices to chose $n_{0}:=\max \left\{n_{1}, n_{2}\right\}$.

The case when there exists a relatively minimal elliptic fibration without multiple fibres $\psi: S \rightarrow \mathbb{P}^{1}$ is of particular interest. Indeed, in this case we have $\kappa(S) \leq 1$, thus $h^{0}\left(S, \mathcal{O}_{S}\left(2 K_{S}-h_{S}\right)\right)=0$ as pointed out in the introduction.

We know that $\mathcal{O}_{S}\left(K_{S}\right) \cong \psi^{*} \mathcal{O}_{\mathbb{P}^{1}}(e-2)$ where $\mathcal{O}_{\mathbb{P}^{1}}(e):=\left(R^{1} \psi_{*} \mathcal{O}_{S}\right)^{\vee}$ (see [7, Theorem V.12.1]): in particular $\kappa(S) \geq 0$ implies $e \geq 2$ and we will assume it from now on, hence $H K_{S} \geq 0$. Moreover, $p_{g}(S)=h^{0}\left(\mathbb{P}^{1}, \mathcal{O}_{\mathbb{P}^{1}}(e-2)\right)$ and $\chi\left(\mathcal{O}_{S}\right)=e$, hence $q(S)=0$ (see [7, Proposition V.12.2] and its proof).

If $\mathcal{O}_{S}(H)$ is very ample and $\mathcal{O}_{S}\left(h_{S}\right):=\mathcal{O}_{S}\left(H+K_{S}\right)$, then $h^{1}\left(S, \mathcal{O}_{S}\left(h_{S}\right)\right)=0$ by the Kodaira vanishing theorem and $\mathcal{O}_{S}\left(h_{S}\right)$ is very ample because $\mathcal{O}_{S}\left(K_{S}\right)$ is globally generated. Finally, $h_{S}^{2}=H^{2}+2 H K_{S}>H K_{S}=h_{S} K_{S}$ because $H K_{S} \geq 0$.

An example of the above set up is the case of an elliptic fibration with a section: in this case there are trivially no multiple fibres. This case has been inspected in [39] with a more direct approach.

In [24] the author proved the existence of special Ulrich bundles of rank 2 on each polarized $K 3$ surface, extending some earlier results (see $[2,18]$ ). The case of Enriques surfaces was examined in $[9,13]$. We list below some other interesting examples.

Example 6.2. Let $S$ be a regular surface and $\mathcal{O}_{S}\left(h_{S}\right) \cong \mathcal{O}_{S}\left(n K_{S}\right)$ for some integer $n \geq 3$. Then $S$ supports a $\mu$-stable special Ulrich bundle of rank 2 with respect to $\mathcal{O}_{S}\left(h_{S}\right)$. 
Indeed, in this case $\mathcal{Z}_{1} \neq \emptyset$ (see [36]) and $K_{S}$ is ample, hence $S$ is minimal: then $h^{1}\left(S, \mathcal{O}_{S}\left(h_{S}\right)\right)=0$ thanks to [7, Proposition VII.5.3]. Moreover, the conditions $h_{S}^{2}>h_{S} K_{S}$ and $h^{0}\left(S, \mathcal{O}_{S}\left(2 K_{S}-h_{S}\right)\right)=0$ are trivially satisfied in this case.

The above result cannot be extended to the cases $1 \leq n \leq 2$ using the same argument. Indeed, $h^{0}\left(S, \mathcal{O}_{S}\left(2 K_{S}-h_{S}\right)\right) \geq 1$ in these cases. In particular, we cannot apply Theorem 5.1 in the base case of the induction in the proof of Theorem 1.1.

Notice that the case $n=2$ could be within reach: indeed it would be sufficient to check that $h^{0}\left(S, \mathcal{F} \otimes \mathcal{I}_{Z \mid S}\right)<h^{0}(S, \mathcal{F})$ in the proof of Proposition 3.3, because $h^{0}\left(S, \mathcal{O}_{S}\left(2 K_{S}-h_{S}\right)\right)=1$ (for some similar results in this direction see Example 8.4).

On the other hand, the case $n=1$ seems to be out of reach with our methods, because $h^{0}\left(S, \mathcal{O}_{S}\left(2 K_{S}-h_{S}\right)\right)=p_{g}(S)$ in this case.

It is classically known (see [11] and the references therein) that non-degenerate surfaces $S$ of degree $d=2 N-2+s$ in $\mathbb{P}^{N}$ are geometrically ruled or $K 3$ when $s \leq 0$. In [11] the author gives a description of surfaces with $1 \leq s \leq N-3$ when $\mathcal{O}_{S}\left(h_{S}\right)$ is non-special. In all these cases $s \equiv h_{S} K_{S}(\bmod 2)$,

$$
h_{S} K_{S} \leq \begin{cases}3 s & \text { if } 0 \leq s \leq N-3 \\ s-2 & \text { if } s<0\end{cases}
$$

Example 6.3. Let $k \cong \mathbb{C}$ and $S \subseteq \mathbb{P}^{N}$ a non-ruled, non-degenerate surface of degree $2 N-2+s$ where $N \geq 3+s$ and $0 \leq s \leq 3$ such that $\mathcal{O}_{S}\left(h_{S}\right)$ is nonspecial. Moreover, if $p_{g}(S) \geq 1$ and $\kappa(S)=2$, we also assume

$$
N \geq \frac{5 s+3}{2}
$$

Then $S$ supports special Ulrich bundles of rank 2 with respect to $\mathcal{O}_{S}\left(h_{S}\right)$. Since $k \cong \mathbb{C}$, it follows that $\mathcal{Z}_{1} \neq \emptyset$ hence such bundles can be taken $\mu$-stable.

The classification given in [11, Propositions 2.1, 2.2, 2.3] and [11, Lemma 1.10] imply that $S$ is always regular in these cases, hence the assertion follows from $[13,14]$ if $p_{g}(S)=0$. Thus, from now on, we will assume $p_{g}(S) \geq 1$. Notice that the inequality $N \geq 3+s$ implies $h_{S}^{2}>h_{S} K_{S}$.

If $\kappa(S) \leq 1$, then $h^{0}\left(S, \mathcal{O}_{S}\left(2 K_{S}-h_{S}\right)\right)=0$ trivially. If $\kappa(S)=2$, then Inequality (6.1) implies $2 h_{S} K_{S}-h_{S}^{2}<0$, hence $h^{0}\left(S, \mathcal{O}_{S}\left(2 K_{S}-h_{S}\right)\right)=0$ thanks to the Nakai criterion. In both the cases the assertion then follows from Theorem 1.1 .

Example 6.4. Let $S \subseteq \mathbb{P}^{N}$ be a non-degenerate regular surface with $\kappa(S) \leq 1$, $N \geq p_{g}(S)-2$ and such that $\mathcal{O}_{S}\left(h_{S}\right)$ is non-special. Then $S$ supports simple special Ulrich bundles of rank 2 with respect to $\mathcal{O}_{S}\left(h_{S}\right)$. If $\mathcal{Z}_{1} \neq \varnothing$ and $N \geq p_{g}(S)+1$ such bundles can be taken $\mu$-stable.

Indeed, in this case, we already know that

$$
h^{0}\left(S, \mathcal{O}_{S}\left(K_{S}-h_{S}\right)\right)=h^{0}\left(S, \mathcal{O}_{S}\left(2 K_{S}-h_{S}\right)\right)=0 .
$$


The inequalities $N \geq p_{g}(S)-2$ and $N \geq p_{g}(S)+1$ are respectively equivalent to $h_{S}^{2}+4 \geq h_{S} K_{S}$ and $h_{S}^{2}>h_{S} K_{S}$. Thus the assertions follows from Theorem 1.1.

As a more concrete example, assume that $k \cong \mathbb{C}$ and let $S \subseteq \mathbb{P}^{4}$ be a surface with $\kappa(S)=1$ and degree $d=7,8$. In [41,42] it is shown that the surfaces $S$ are exactly the ones linked to a plane in either a quadro-quartic or a cubo-cubic complete intersection inside $\mathbb{P}^{4}$.

The results in [44] imply that the minimal free resolutions of $\mathcal{I}_{S \mid \mathbb{P}^{4}}$ look like

$$
\begin{aligned}
& 0 \longrightarrow \mathcal{O}_{\mathbb{P}^{4}}(-5)^{\oplus 2} \longrightarrow \mathcal{O}_{\mathbb{P}^{4}}(-2) \oplus \mathcal{O}_{\mathbb{P}^{4}}(-4)^{\oplus 2} \longrightarrow \mathcal{I}_{S \mid \mathbb{P}^{4}} \longrightarrow 0, \\
& 0 \longrightarrow \mathcal{O}_{\mathbb{P}^{4}}(-5)^{\oplus 2} \longrightarrow \mathcal{O}_{\mathbb{P}^{4}}(-3)^{\oplus 2} \oplus \mathcal{O}_{\mathbb{P}^{4}}(-4) \longrightarrow \mathcal{I}_{S \mid \mathbb{P}^{4}} \longrightarrow 0
\end{aligned}
$$

respectively. The cohomology of the exact sequence

$$
0 \longrightarrow \mathcal{I}_{S \mid \mathbb{P}^{4}} \longrightarrow \mathcal{O}_{\mathbb{P}^{4}} \longrightarrow \mathcal{O}_{S} \longrightarrow 0
$$

implies that $\mathcal{O}_{S}\left(h_{S}\right)$ is non-special, $q(S)=0, p_{g}(S)=2$.

In both the cases $S$ is determinantal. Nevertheless, $S$ is not defined by a matrix with linear entries, hence we cannot use the results proved in [31,32] for deducing the existence of an Ulrich bundle.

Similarly, we cannot use the results in [39]. Indeed, in [35, Theorem III.4.2 and Observation III.3.5], the author proves that for a surface $S$ as above which is also very general, then $\operatorname{Pic}(S)$ is generated by $h_{S}$ and $K_{S}$. If the canonical map $\psi: S \rightarrow \mathbb{P}^{1}$ has a section $\sigma: \mathbb{P}^{1} \rightarrow S$, then $C:=\operatorname{im}(\sigma)$ is a rational curve linearly equivalent to $x h_{S}+y K_{S}$ for some integers $x, y$. Since $C$ is the image of a section of the canonical map, it follows that $1=C K_{S}=x h_{S} K_{S}$ which is impossible, because the last number is a multiple of 3 or 4 (according with the two cases $h_{S}^{2}=7,8$ ).

\section{Ulrich-wildness}

In this very short section we deal with the size of the families of Ulrich bundles supported on the surfaces we are interested in.

A variety $X$ is called Ulrich-wild if it supports families of dimension $p$ of pairwise non-isomorphic, indecomposable, Ulrich sheaves with respect to $\mathcal{O}_{X}\left(h_{X}\right)$ for arbitrary large $p$.

Lemma 7.1. Let $X$ be a smooth variety endowed with a very ample line bundle $\mathcal{O}_{X}\left(h_{X}\right)$.

If $\mathcal{E}$ is a simple Ulrich bundle on $X$ such that $\operatorname{dim}_{\operatorname{Ext}_{S}^{1}}(\mathcal{E}, \mathcal{E}) \geq 3$, then $X$ is Ulrich-wild.

Proof. It is an immediate consequence of [26, Theorem A, Corollary 2.1 and Remark $1.6 \mathrm{v})$ ], because every Ulrich bundle is semistable by [12, Theorem 2.9] and each non-zero automorphism of a simple sheaf is an isomorphism.

We deduce the following criterion for the surfaces we are interested in. 
Proposition 7.2. Let $S$ be a surface with $q(S)=0, p_{g}(S) \geq 1$, endowed with a very ample non-special line bundle $\mathcal{O}_{S}\left(h_{S}\right)$. Assume $h^{0}\left(S, \mathcal{O}_{S}\left(2 K_{S}-h_{S}\right)\right)=0$ and $h_{S}^{2}+4 \geq h_{S} K_{S}$.

Then $S$ is Ulrich-wild.

Proof. Consider the bundle $\mathcal{F}$ defined in Construction (3.1). We know from Proposition 3.3 that $\mathcal{F}$ is simple and $\operatorname{dim}_{\operatorname{Ext}_{S}^{1}}^{1}(\mathcal{F}, \mathcal{F})=p_{g}(S)$. Then, Equality (2.3) yields

$$
h_{S}^{2}-K_{S}^{2}+\chi\left(\mathcal{O}_{S}\right)+4=1+p_{g}(S)-\chi\left(\mathcal{F} \otimes \mathcal{F}^{\vee}\right)=\operatorname{dim} \operatorname{Ext}_{S}^{1}(\mathcal{F}, \mathcal{F}) \geq 0 .
$$

The same argument applied to the Ulrich bundle $\mathcal{E}$ defined in Theorem 1.1, then yields

$$
\operatorname{dim} \operatorname{Ext}_{S}^{1}(\mathcal{E}, \mathcal{E})=1+p_{g}(S)-\chi\left(\mathcal{E} \otimes \mathcal{E}^{\vee}\right)=h_{S}^{2}-K_{S}^{2}+5 \chi\left(\mathcal{O}_{S}\right) \geq 3 .
$$

Thus the statement follows immediately from Lemma 7.1.

The above proposition extends [13, Theorem 1.3] to the case $p_{g}(S) \geq 1$.

Corollary 7.3. Let $S$ be one of the surfaces described in Examples 6.1, 6.2, 6.3, 6.4 .

Then $S$ is Ulrich-wild.

Proof. The statement is a trivial consequence of Proposition 7.2.

\section{Ulrich bundles on surfaces of low degree}

In [15, Theorems 1.4 and 1.5] the author deals with the existence of special Ulrich bundles on surfaces of low degree on surfaces $S \subseteq \mathbb{P}^{N}$.

We start this section by improving [15, Theorem 1.4]. We work over the complex field $\mathbb{C}$, hence $S_{0}$ is dense for each surface $S$ with $\kappa(S) \geq 0$ by Remark 2.3 .

Theorem 8.1. Let $k \cong \mathbb{C}, S \subseteq \mathbb{P}^{N}$ a surface of degree $d \leq 8$.

Then the following assertions holds.

(1) If $\kappa(S) \leq 1$, then $S$ supports special Ulrich bundles.

(2) If $\kappa(S)=2$ and $S$ is general, then $S$ supports special Ulrich bundles.

Proof. Thanks to [15, Theorem 1.4] we know that if $\kappa(S) \neq 1$, then $S$ supports Ulrich bundles $\mathcal{E}$ of even rank $r_{U l r i c h}^{s p}$ as in [15, Table A] and such that

$$
c_{1}(\mathcal{E})=\frac{r_{\text {Ulrich }}^{s p}}{2}\left(3 h_{S}+K_{S}\right) .
$$

In particular $r_{\text {Ulrich }}^{s p}=2$ if either $\kappa(S) \leq 0$ or $\kappa(S)=2$ and $S$ is general. Thus such an Ulrich bundle $\mathcal{E}$ is special in these cases.

If $\kappa(S)=1$, taking into account of the classification in [15, Table A] and the results in [41,42], we know that $S \subseteq \mathbb{P}^{4}$ is a properly elliptic surface of degree either 7 or 8 . The existence of special Ulrich bundles on such surfaces has been proved in Example 6.4. 
We now extend [15, Theorem 1.5]. Here $\pi(S)$ denotes the genus of a general plane section of $S$.

Theorem 8.2. Let $k \cong \mathbb{C}, S \subseteq \mathbb{P}^{N}$ a surface of degree $d \leq 8$.

Then $S$ is Ulrich-wild if and only if either $d \geq 5$, or $d \leq 4$ and $\pi(S) \geq 1$.

Proof. The statement coincides with [15, Theorem 1.5] when $\kappa(S) \neq 1$.

If $\kappa(S)=1$, then we know (see the proof of Theorem 8.1) that $S$ is regular, non-special, $h_{S}^{2}+4 \geq h_{S} K_{S}$ and $h^{0}\left(S, \mathcal{O}_{S}\left(2 K_{S}-h_{S}\right)\right)=0$. Then the statement follows from Proposition 7.2.

We close the section with some examples dealing with non-degenerate surfaces of degree $d>8$ in $\mathbb{P}^{4}$.

Example 8.3. Let $k \cong \mathbb{C}$ and $S \subseteq \mathbb{P}^{4}$ a non-degenerate surface of degree 9 with non-special $\mathcal{O}_{S}\left(h_{S}\right)$ and $h_{S}^{2}>h_{S} K_{S}$.

The surfaces as above are regular, thanks to [4, Theorem 0.1]. If $p_{g}(S)=0$, then $S$ supports special Ulrich bundles which are $\mu$-stable thanks to [13,14], because $S$ is neither a rational normal scroll nor a plane by degree reasons. In what follows we will briefly deal with the case $p_{g}(S) \geq 1$.

We have $h^{2}\left(S, \mathcal{O}_{S}\left(h_{S}\right)\right)=0$ because $9=h_{S}^{2}>h_{S} K_{S}$. The so-called Severi Theorem (see [46]) implies $h^{0}\left(S, \mathcal{O}_{S}\left(h_{S}\right)\right)=5$. Since $\mathcal{O}_{S}\left(h_{S}\right)$ is non-special, it follows from Equality 2.3 that $h_{S} K_{S}=2 \chi\left(\mathcal{O}_{S}\right)-1$. Moreover, the adjunction formula returns $h_{S} K_{S}=2 \pi(S)-11$. By combining such two equalities we obtain $\chi\left(\mathcal{O}_{S}\right)=\pi(S)-5$. Finally, the inequality $9>h_{S} K_{S}$ forces $\pi(S) \leq 9$.

Thanks to the above discussion and to the classification in [4, Theorem 0.1 and its proof] we have to deal only with the following cases.

- A minimal properly elliptic surface $S$ with $h_{S} K_{S}=3, K_{S}^{2}=0, p_{g}(S)=1$.

- A minimal surface $S$ of general type with $h_{S} K_{S}=5, K_{S}^{2}=1, p_{g}(S)=2$.

- A surface $S$ linked with a possibly singular/reducible cubic scroll $Y$ via a cuboquartic complete intersection: in this case $h_{S} K_{S}=7, K_{S}^{2}=2, p_{g}(S)=3$.

In the first case case we trivially have $h^{0}\left(S, \mathcal{O}_{S}\left(2 K_{S}-h_{S}\right)\right)=0$, hence Theorem 1.1 yields the existence of $\mu$-stable special Ulrich bundles on $S$.

In the remaining cases the vanishing $h^{0}\left(S, \mathcal{O}_{S}\left(2 K_{S}-h_{S}\right)\right)=0$ is not evident as above. E.g., let us examine the second case. If $h^{0}\left(S, \mathcal{O}_{S}\left(2 K_{S}-h_{S}\right)\right) \neq 0$, then $h^{0}\left(S, \mathcal{O}_{S}\left(2 K_{S}\right)\right) \geq h^{0}\left(S, \mathcal{O}_{S}\left(h_{S}\right)\right)=5$. Moreover, $h^{2}\left(S, \mathcal{O}_{S}\left(2 K_{S}\right)\right)=0$ by the Serre duality. Thus Equality (2.3) implies

$$
h^{0}\left(S, \mathcal{O}_{S}\left(2 K_{S}\right)\right)-h^{1}\left(S, \mathcal{O}_{S}\left(2 K_{S}\right)\right)=K_{S}^{2}+\chi\left(\mathcal{O}_{S}\right) .
$$

It follows that $h^{1}\left(S, \mathcal{O}_{S}\left(2 K_{S}\right)\right) \geq 1$, hence $S$ would not be minimal thanks to [7, Corollary VII.5.4], a contradiction. Thus $h^{0}\left(S, \mathcal{O}_{S}\left(2 K_{S}-h_{S}\right)\right)=0$, hence the existence of $\mu$-stable special Ulrich bundles on $S$ still follows from Theorem 1.1.

In the third case the same argument does not hold. Indeed $\kappa(S) \geq 1$ and $K_{S}^{2}=2$. The minimal model $S_{\text {min }}$ of $S$ satisfies $K_{S_{\text {min }}}^{2} \geq 2$, thus $\kappa(S)=2$. Nevertheless, the vanishing $h^{0}\left(S, \mathcal{O}_{S}\left(2 K_{S}-h_{S}\right)\right)=0$ can be proved also in this case. Indeed, 
in [4, Section (2.13)] the authors show that the general hyperplane section $C$ of the surface $Y$ linked to $S$ is aCM with $p_{a}(C)=0$. This fact and the Riemann-Roch theorem on $C$ implies that the ideal of $C$, hence of $Y$, is generated by the minors of a $3 \times 2$ matrix of linear forms. Thus there exists an exact sequence of the form

$$
0 \longrightarrow \mathcal{O}_{\mathbb{P}^{4}}(-3)^{\oplus 2} \longrightarrow \mathcal{O}_{\mathbb{P}^{4}}(-2)^{\oplus 3} \longrightarrow \mathcal{I}_{Y \mid \mathbb{P}^{4}} \longrightarrow 0 .
$$

Thanks to [44] there is a resolution of the form

$$
0 \longrightarrow \mathcal{O}_{\mathbb{P}^{4}}(-5)^{\oplus 3} \longrightarrow \mathcal{O}_{\mathbb{P}^{4}}(-3) \oplus \mathcal{O}_{\mathbb{P}^{4}}(-4)^{\oplus 3} \longrightarrow \mathcal{I}_{X \mid \mathbb{P}^{4}} \longrightarrow 0 .
$$

Thus [20, Proposition II.2.4] implies $h^{0}\left(S, \mathcal{O}_{S}\left(2 K_{S}-h_{S}\right)\right)=0$, hence Theorem 1.1 yields the existence of $\mu$-stable special Ulrich bundles on $S$.

Example 8.4. Let $k \cong \mathbb{C}$ and $S \subseteq \mathbb{P}^{4}$ a non-degenerate surface of degree 10 with non-special $\mathcal{O}_{S}\left(h_{S}\right)$ and $h_{S}^{2}>h_{S} K_{S}$.

Surfaces as above are classified in [45, Theorem 0.1]. If $q(S)=p_{g}(S)=0$, then $S$ is not a rational normal scroll by degree reasons, hence it supports special Ulrich bundles which are $\mu$-stable from $[13,14]$.

From now on we will assume that $q(S)$ and $p_{g}(S)$ do not vanish simultaneously. The argument of Example 8.3 yields $h_{S} K_{S}=2 \chi\left(\mathcal{O}_{S}\right)$ and $h_{S} K_{S}=2 \pi(S)-12$, hence $\chi\left(\mathcal{O}_{S}\right)=\pi(S)-6$ and $\pi(S) \leq 10$. Thus the results in [45, Theorem 0.1 and its proof in Section 9] lead us to deal only with the following cases.

- An abelian surface.

- A bielliptic surface.

- A minimal surface $S$ of general type with $h_{S} K_{S}=6, K_{S}^{2}=3, p_{g}(S)=2$ : in this case $\mathcal{O}_{S}\left(h_{S}\right) \cong \mathcal{O}_{S}\left(2 K_{S}-A\right)$, where $A$ is a curve such that $A^{2}=-2$ and $A K_{S}=0$.

- A minimal surface $S$ of general type with $h_{S} K_{S}=8, K_{S}^{2}=4, q(S)=0$, $p_{g}(S)=3:$ in this case $\mathcal{O}_{S}\left(h_{S}\right) \cong \mathcal{O}_{S}\left(2 K_{S}-A_{1}-A_{2}-A_{3}\right)$, where the $A_{i}$ 's are disjoint curves such that $A_{i}^{2}=-2$ and $A_{i} K_{S}=0$.

The existence of special Ulrich bundles on Abelian or bielliptic surfaces has been proved in $[8,10]$. Such bundles can be taken $\mu$-stable: see [8] for abelian surfaces and $[16,17]$ for bielliptic surfaces.

In the other cases $h^{0}\left(S, \mathcal{O}_{S}\left(2 K_{S}-h_{S}\right)\right)=1$, hence Theorem 1.1 does not give any information on the existence of special Ulrich bundles on such an $S$.

Example 8.5. Let $k \cong \mathbb{C}$ and $S \subseteq \mathbb{P}^{4}$ a non-degenerate surface of degree $d \geq 11$ with $\mathcal{O}_{S}\left(h_{S}\right)$ non-special and $h_{S}^{2}>h_{S} K_{S}$.

In [33, Theorems 1 and 2] and [37, Proposition] the authors classify non-special and non-degenerate surfaces $S \subseteq \mathbb{P}^{4}$ which are not of general type. As a by-product they show that such surfaces have degree at most 10 . Thus $S$ is necessarily a surface of general type, hence $\chi\left(\mathcal{O}_{S}\right) \geq 1$. Equality (2.3), the vanishings

$$
h^{0}\left(S, \mathcal{O}_{S}\left(K_{S}-h_{S}\right)\right)=0, \quad h^{1}\left(S, \mathcal{O}_{S}\left(K_{S}-h_{S}\right)\right)=h^{1}\left(S, \mathcal{O}_{S}\left(h_{S}\right)\right)=0
$$

and the equality $h^{0}\left(S, \mathcal{O}_{S}\left(h_{S}\right)\right)=5$ (see [46]) imply that

$$
h_{S} K_{S}=2 \chi\left(\mathcal{O}_{S}\right)+d-10 \geq 3:
$$


hence the condition $h_{S} K_{S}<h_{S}^{2}=d$ yields $\chi\left(\mathcal{O}_{S}\right) \leq 4$. The double point formula (see [28], Example A.4.1.3) is

$$
K_{S}^{2}=\chi\left(\mathcal{O}_{S}\right)+25+\frac{d(d-15)}{2} .
$$

The Hodge index theorem for the divisors $h_{S}, K_{S}$, Equality (8.2) and the hypothesis $h_{S} K_{S}<d$ then yields $d \leq 12$.

Taking into account the bound $1 \leq \chi\left(\mathcal{O}_{S}\right) \leq 4$, computing $K_{S}^{2}, h_{S} K_{S}$, applying again the Hodge index theorem for the divisors $h_{S}, K_{S}$ and Equality (8.2) one easily checks that the case $d=12$ cannot occur. If $d=11$, the same argument yields the $\chi\left(\mathcal{O}_{S}\right)=4, K_{S}^{2}=7, h_{S} K_{S}=9$. If $S$ is not minimal, then $K_{S}=K_{0}+E$ where $K_{0} E=0, K_{0}^{2} \geq 8$ and $h_{S} K_{0} \leq 8$. The Hodge index theorem for the divisors $h_{S}$, $K_{0}$, then yields a contradiction. It follows that $S$ is minimal. If $q(S) \geq 1$, then $p_{g}(S) \geq 4$, hence we should have $K_{S}^{2} \geq 8$, thanks to [21, Théorè eme 6.1], a contradiction. We deduce that $S$ is minimal and regular.

We do not know if such a surface exists. Anyhow, if it exists, let $C \in\left|K_{S}\right|$ : we have $\operatorname{deg}(C)=h_{S} K_{S}=9$ and $p_{a}(C)=K_{S}^{2}+1=8$. The cohomology of the exact sequence

$$
0 \longrightarrow \mathcal{O}_{S}(-C) \longrightarrow \mathcal{O}_{S} \longrightarrow \mathcal{O}_{C} \longrightarrow 0,
$$

tensored by $\mathcal{O}_{S}\left(2 K_{S}-h_{S}\right)$, Equalities (8.1) and the Riemann-Roch theorem on $C$ imply

$$
\begin{aligned}
h^{0}\left(S, \mathcal{O}_{S}\left(2 K_{S}-h_{S}\right)\right) & =h^{0}\left(C, \mathcal{O}_{C} \otimes \mathcal{O}_{S}\left(2 K_{S}-h_{S}\right)\right) \\
& =h^{0}\left(C, \mathcal{O}_{C}\left(K_{C}\right) \otimes \mathcal{O}_{S}\left(h_{S}-2 K_{S}\right)\right)-2 .
\end{aligned}
$$

The adjunction formula on $S$ returns $\mathcal{O}_{C}\left(K_{C}\right) \cong \mathcal{O}_{C} \otimes \mathcal{O}_{S}\left(2 K_{S}\right)$, hence

$$
h^{0}\left(S, \mathcal{O}_{S}\left(2 K_{S}-h_{S}\right)\right)=h^{0}\left(C, \mathcal{O}_{C}\left(h_{C}\right)\right)-2
$$

Thus $h^{0}\left(C, \mathcal{O}_{C}\left(h_{C}\right)\right) \geq 2$. If $h^{0}\left(C, \mathcal{O}_{C}\left(h_{C}\right)\right)=2$, then the cohomology of Sequence (8.3) tensored by $\mathcal{O}_{S}\left(h_{S}\right)$ would imply that $C$ is contained in three linearly independent hyperplanes of $\mathbb{P}^{4}$, hence $C$ would be a line, a contradiction. We deduce that $3 \leq h^{0}\left(C, \mathcal{O}_{C}\left(h_{C}\right)\right)$, hence $1 \leq h^{0}\left(S, \mathcal{O}_{S}\left(2 K_{S}-h_{S}\right)\right)$ : again Theorem 1.1 does not give any information on Ulrich bundles.

Acknowledgements I would like to express my thanks to D. Faenzi and A.F. Lopez for many helpful discussions and suggestions about the content of the present paper. I am particularly indebted with the referee for her/his criticisms, questions, remarks and suggestions which have considerably improved the whole exposition.

Funding Open access funding provided by Politecnico di Torino within the CRUI-CARE Agreement.

Open Access This article is licensed under a Creative Commons Attribution 4.0 International License, which permits use, sharing, adaptation, distribution and reproduction in any medium or format, as long as you give appropriate credit to the original author(s) and the source, provide a link to the Creative Commons licence, and indicate if changes were made. 
The images or other third party material in this article are included in the article's Creative Commons licence, unless indicated otherwise in a credit line to the material. If material is not included in the article's Creative Commons licence and your intended use is not permitted by statutory regulation or exceeds the permitted use, you will need to obtain permission directly from the copyright holder. To view a copy of this licence, visit http://creativecommons.org/ licenses/by/4.0/.

\section{References}

[1] Altman, A.B., Kleiman, S.L.: Compactifying the Picard scheme. Adv. Math. 35, 50-112 (1980)

[2] Aprodu, M., Farkas, G., Ortega, A.: Minimal resolutions, Chow forms and Ulrich bundles on $K 3$ surfaces. J. Reine Angew. Math. 730, 225-249 (2017)

[3] Artamkin, I.V. Deformation of torsion-free sheaves on an algebraic surface. (Russian) Izv. Akad. Nauk SSSR Ser. Mat. 54, : 435-468; translation in Math. USSR-Izv. 36(1991), 449-485 (1990)

[4] Aure, A.B., Ranestad, K.: The smooth surfaces of degree 9 in $\mathbb{P}^{4}$. In 'Complex projective geometry' (Trieste, 1989/Bergen, 1989), pp. 32-46. L.M.S. Lecture Note Ser., 179, Cambridge U.P. (1992)

[5] Baragar, A., McKinnon, D.: K3 surfaces, rational curves, and rational points. J. Number Theory 130, 1470-1479 (2010)

[6] Baran, A.: Duality for the hyperext on complex spaces. Rev. Roumaine Math. Pures Appl. 31, 95-120 (1986). Bucharest

[7] Barth, W., Hulek, K., Peters, Ch., van de Ven, A.: Compact Complex Surfaces, 2nd edn., xii+436 p. Springer, Berlin (2004)

[8] Beauville, A.: Ulrich bundles on abelian surfaces. Proc. Am. Math. Soc. 144, 46094611 (2016)

[9] A. Beauville: Ulrich bundles on surfaces with $p_{g}=q=0$. Available at arXiv:1607.00895 [math.AG]

[10] Beauville, A.: An introduction to Ulrich bundles. Eur. J. Math. 4, 26-36 (2018)

[11] Buium, A.: On surfaces of degree at most $2 n+1$ in $\mathbf{P}^{n}$. In 'Algebraic geometry, Bucharest 1982 (Bucharest, 1982)', pp. 47-67. L.N.M. 1056, 47-67 (1984)

[12] Casanellas, M., Hartshorne, R., Geiss, F., Schreyer, F.O.: Stable Ulrich bundles. Int. J. Math. 23, 1250083 (2012)

[13] Casnati, G.: Special Ulrich bundles on non-special surfaces with $p_{g}=q=0$. Int. J. Math. 28, 1750061 (2017)

[14] Casnati, G.: Erratum "Special Ulrich bundles on non-special surfaces with $p_{g}=q=$ 0”. Int. J. Math. 29, 1892001 (2018)

[15] Casnati, G.: Examples of surfaces which are Ulrich-wild. To appear in P. Am. Math. Soc

[16] Casnati, G.: Ulrich bundles on non-special surfaces with $p_{g}=0$ and $q=1$. Rev. Mat. Complut. 32, 559-574 (2019)

[17] Casnati, G.: Correction to: Ulrich bundles on non-special surfaces with $p_{g}=0$ and $q=1$. Rev. Mat. Complut. 32, 575-577 (2019)

[18] Coskun, E., Kulkarni, R.S., Mustopa, Y.: Pfaffian quartic surfaces and representations of Clifford algebras. Doc. Math. 17, 1003-1028 (2012)

[19] Cossec, F., Dolgachev, I.: Smooth rational curves on Enriques surfaces. Math. Ann. 272, 369-384 (1985) 
[20] D'Ambra, M.: Partially ample divisors on projective schemes and Ulrich vector bundles on projectively Cohen-Macaulay surfaces in $\mathbb{P}^{4}$. Ph.D. thesis, Università degli Studi di Roma - Tor Vergata, vii+85 p. (2020)

[21] Debarre, O.: Inégalités numériques pour les surfaces de type général. Appendice: 'L'inégalité $p_{g} \geq 2 q-4$ pour les surfaces de type général' par A. Beauville. Bull. Soc. Math. France 110(3), 319-346 (1982)

[22] Eisenbud, D., Herzog, J.: The classification of homogeneous Cohen-Macaulay rings of finite representation type. Math. Ann. 280, 347-352 (1988)

[23] Eisenbud, D., Schreyer, F.O., Weyman, J.: Resultants and Chow forms via exterior syzigies. J. Am. Math. Soc. 16, 537-579 (2003)

[24] Faenzi, D.: Ulrich sheaves on $K 3$ surfaces. Algebra Number Theory 13, 1443-1454 (2019)

[25] Faenzi, D., Malaspina, F.: Surfaces of minimal degree of tame representation type and mutations of Cohen-Macaulay modules. Adv. Math. 310, 663-695 (2017)

[26] Faenzi, D., Pons-Llopis, J.: The CM representation type of projective varieties. Available at arXiv:1504.03819v2 [math.AG]

[27] Fulton, W.: Intersection Theory, xiv+470 p. Springer, Ergebnisse der Mathematik und ihrer Grenzgebiete (1984)

[28] Hartshorne, R.: Algebraic Geometry, G.T.M. 52, xvi+496 p. Springer, Berlin (1977)

[29] Hartshorne, R.: Coherent functors. Adv. Math. 140, 44-94 (1998)

[30] Huybrechts, D., Lehn, M.: The Geometry of Moduli Spaces of Sheaves, 2nd edn., xviii+325 p. Cambridge Mathematical Library, Cambridge (2010)

[31] Kleppe, J.O., Miró-Roig, R.M.: On the normal sheaf of determinantal varieties. J. Reine Angew. Math. 719, 173-209 (2016)

[32] Kleppe, J.O., Miró-Roig, R.M.: The representation type of determinantal varieties. Algebr. Represent. Theory 20, 1029-1059 (2017)

[33] Ida, M., Mezzetti, E.: Smooth non-special surfaces in $\mathbb{P}^{4}$. Manuscripta Math. 68, 57-77 (1990)

[34] Lazarsfeld, R.: Positivity in Algebraic Geometry. I. Classical Setting: Line Bundles and Linear Series. A Series of Modern Surveys in Mathematics, 48, xviii+387 p. Springer, Berlin (2004)

[35] Lopez, A.: Noether-Lefschetz theory and the Picard group of projective surfaces. Memoirs Am. Math. Soc. 89, x+100 (1991)

[36] Lu, S.S.Y., Miyaoka, Y.: Bounding curves in algebraic surfaces by genus and Chern numbers. Math. Res. Lett. 2, 663-676 (1995)

[37] Mezzetti, E., Ranestad, K.: The non-existence of a smooth sectionally non-special surface of degree 11 and sectional genus 8 in the projective fourspace. Manuscripta Math. 70, 279-283 (1991)

[38] Miró-Roig, R.M.: The representation type of rational normal scrolls. Rend. Circ. Mat. Palermo 62, 153-164 (2013)

[39] Miró-Roig, R.M., Pons-Llopis, J.: Special Ulrich bundles on regular Weierstrass fibrations. Math. Z. 293, 1431-1441 (2019)

[40] Mukai, S.: Symplectic structure of the moduli space of sheaves on an abelian or $K 3$ surface. Invent. Math. 77, 101-116 (1984)

[41] Okonek, Ch.: Über 2-codimensionale Untermannigfaltigkeiten vom Grad 7 in $\mathbb{P}^{4}$ und $\mathbb{P}^{5}$. Math. Z. 187, 209-219 (1984)

[42] Okonek, Ch.: Flächen vom Grad 8 im $\mathbb{P}^{4}$. Math. Z. 191, 207-223 (1986)

[43] Okonek, C., Schneider, M., Spindler, H.: Vector bundles on complex projective spaces. Progress in Mathematics 3, vii+389 (1980)

[44] Peskine, C., Szpiro, L.: Liaison des variétés algébriques. I. Inv. Math. 26, 271-302 (1974) 
[45] Ranestad, K.: On smooth surfaces of degree ten in the projective fourspace, $107 \mathrm{p}$. thesis, Oslo, (1988)

[46] Severi, F.: Intorno ai punti doppi impropri di una superficie generale dello spazio a quattro dimensioni, e a' suoi punti tripli apparenti. Rend. Circ. Mat. Palermo 15, 33-51 (1901)

Publisher's Note Springer Nature remains neutral with regard to jurisdictional claims in published maps and institutional affiliations. 